\title{
Les modifications apportées à la Loi sur les normes du travail du Québec
}

\section{Gilles Trudeau}

\section{Q OpenEdition}

1 Journals

\section{Édition électronique}

URL : https://journals.openedition.org/rdctss/1678

DOI : $10.4000 /$ rdctss. 1678

ISSN : 2262-9815

Éditeur

Centre de droit comparé du travail et de la sécurité sociale

\section{Édition imprimée}

Date de publication : 1 avril 2019

Pagination : 184-187

ISSN : 2117-4350

\section{Référence électronique}

Gilles Trudeau, "Les modifications apportées à la Loi sur les normes du travail du Québec », Revue de droit comparé du travail et de la sécurité sociale [En ligne], 1 | 2019, mis en ligne le 01 novembre 2021, consulté le 13 novembre 2021. URL : http://journals.openedition.org/rdctss/1678 ; DOI : https:// doi.org/10.4000/rdctss. 1678

\section{(c) (†) $९$}

Revue de droit comparé du travail et de la sécurité sociale est mise à disposition selon les termes de la Licence Creative Commons Attribution - Pas d'Utilisation Commerciale - Pas de Modification 4.0 International. 


\section{GILLES TRUDEAU}

UNIVERSITÉ DE MONTRÉAL

\section{LES MODIFICATIONS APPORTÉES À LA LOI SUR LES NORMES DU TRAVAIL DU QUÉBEC} à la Loi sur les normes du travail ${ }^{1}$ du Québec figurent certainement parmi les plus significatifs. Proposant des mesures favorisant la conciliation travail-famille et s'intéressant aux salariés parmi les plus vulnérables, ils reflètent les évolutions profondes que connaissent la composition de la main-d'œuvre et l'organisation du travail.

\section{I - LA LOI SUR LES NORMES DU TRAVAIL, SON CONTENU ET SES EFFETS}

Au sein de la fédération canadienne, la compétence constitutionnelle relative au droit du travail appartient principalement aux provinces. Chacune est souveraine en la matière, et peut ainsi définir son droit du travail comme elle l'entend. Celui-ci est toutefois passablement le même d'une province à l'autre, procédant essentiellement des trois mêmes grandes sources: le droit commun et le contrat individuel de travail, la négociation collective et la législation relative aux conditions de travail.

La Loi sur les normes du travail, dont la première mouture a été adoptée en 1979 par le législateur québécois, relève de la dernière source, et représente indéniablement l'exemple d'une intervention importante de l'État dans la définition des conditions de travail, du moins à l'échelle nord-américaine. Les normes minimales qu'elle prescrit s'imposent comme des conditions de travail en deçà desquelles aucun travail salarié ne saurait être permis. Ses articles 93 et 94 le corroborent, indiquant que les normes minimales du travail sont d'ordre public, et que les parties à la négociation collective ou au contrat individuel de travail ne peuvent librement établir que des conditions de travail plus avantageuses. Compte tenu de son objectif fondamental, la Loi sur les normes du travail vise l'ensemble de la main-d'œuvre salariée, s'appliquant pratiquement à tous les salariés qui travaillent au Québec, à quelques exceptions près.

La Loi sur les normes du travail exerce avant tout une fonction protectrice en assurant aux travailleurs salariés des conditions minimales de travail que les mécanismes du libre marché ne permettraient pas aux plus vulnérables d'obtenir. Elle vise à ce titre les conditions de travail les plus centrales comme le salaire, les heures de travail, le congé annuel et les jours fériés. Ce sont les normes les plus anciennes. Plus récemment toutefois, la Loi a aussi servi de véhicule à la mise en œuvre de politiques gouvernementales d'application universelle. Ainsi sont apparues des normes relatives à l'équilibre travail-famille, à la protection de l'emploi ou encore à l'élimination du harcèlement psychologique ou sexuel. Les modifications apportées à la Loi en 2018, tout en s'inscrivant dans cette dernière perspective, visent aussi à protéger plus adéquatement certaines

1 RLRQ, c. N-1-1, ci-après la [LNT]. 
catégories de travailleurs que les nouvelles formes d'organisation du travail ont rendues particulièrement vulnérables².

\section{II - LES MODIFICATIONS DU TRAVAIL EFFECTUÉ PAR L'INTERMÉDIAIRE D'UNE AGENCE DE PLACEMENT}

Le recours aux agences de placement pour combler les besoins en main-d'œuvre contribue à exacerber la précarité de la situation d'un nombre toujours croissant de travailleurs. Généralement considérée comme l'employeur légal des salariés qu'elle embauche, l'agence érige un écran entre ceux-ci et les organisations clientes dans lesquelles ils sont affectés. Ces dernières bénéficient ainsi de leurs services, tout en évitant les obligations dont un employeur serait normalement imputable à leur égard. Les récents amendements traitent la situation de diverses manières. D'une part, ils assujettissent l'exploitation d'une agence de placement à l'obtention d'un permis émis par la CNESST ${ }^{3}$, et précisent qu'une entreprise cliente ne pourra retenir les services d'une agence non titulaire d'un tel permis. Les conditions d'obtention du permis et les obligations qui y sont rattachées seront établies par un règlement du gouvernement dont la teneur n'était pas encore connue au moment d'écrire ces lignes. On peut toutefois espérer que le permis ne sera octroyé qu'aux seules agences qui offrent des garanties suffisantes de conformité aux obligations légales qu'elles doivent assumer à titre d'employeurs des salariés qu'elles recrutent. Ce règlement devra aussi départager les obligations qui incombent à l'agence de celles qui incombent à l'entreprise cliente. D'autre part, les amendements prescrivent que l'agence et l'entreprise cliente sont solidairement responsables des obligations pécuniaires fixées par la Loi ou par règlement. L'entreprise cliente aura ainsi avantage à s'assurer que l'agence respecte les obligations pécuniaires que la Loi lui impose, à défaut de quoi elle en sera elle-même la débitrice envers les salariés de l'agence dont elle utilise les services. De plus, est dorénavant prohibée toute différence dans le taux de salaire défavorable à un salarié, relativement à celui accordé aux autres salariés qui exécutent le même travail dans le même établissement, uniquement en raison du fait que ses services sont retenus par l'intermédiaire d'une agence de placement. Ce faisant, la Loi prive l'entreprise cliente d'un avantage qui, dans certains cas, l'aurait incitée à recruter une partie de son personnel de cette façon.

\section{III - LES TRAVAILLEURS ÉTRANGERS TEMPORAIRES}

Les travailleurs étrangers temporaires, auxquels le recours a largement augmenté au cours des dernières décennies au Québec, particulièrement dans les domaines agricole et du travail domestique, ont aussi attiré l'attention du législateur. Même s'ils sont formellement assujettis à la Loi sur les normes du travail, plusieurs abus commis à leur endroit quant à leurs conditions de travail et d'hébergement ont été dénoncés. Quatre dispositions les visant spécifiquement ont été insérées dans la Loi en 2018. Ainsi, tout employeur qui embauche un travailleur étranger temporaire doit dorénavant informer la CNESST de la date de son arrivée, de la durée de son contrat ainsi que, s'il repart prématurément, de la date et des raisons de son départ. La CNESST peut de plus exercer tout recours judiciaire pour le compte d'un travailleur étranger temporaire, même sans plainte de sa part, lorsqu'après enquête, elle croit que celui-ci a été victime d'une atteinte à un droit que la Loi lui confère. L'employeur ne peut exiger d'un tel travailleur qu'il

2 Ces modifications résultent de la Loi modifiant la Loi sur les normes du travail et d'autres dispositions législatives afin principalement de faciliter la conciliation famille-travail, L.Q. 2018, chap. 21, adoptée le 12 juin 2018.

3 La Commission des normes, de l'équité, de la santé et de la sécurité du travail, l'agence gouvernementale chargée de l'application de la Loi, ci-après la [CNESST]. 
lui confie la garde de documents personnels, comme son passeport par exemple, ni de biens lui appartenant. L'employeur ne peut non plus exiger du travailleur étranger temporaire des frais de recrutement autres que ceux autorisés en application d'un programme gouvernemental canadien. Enfin, les agences auxquelles les employeurs recourent pour retenir les services de travailleurs étrangers temporaires sont dorénavant requises d'obtenir un permis, comme c'est le cas des agences de placement.

\section{IV - LA DISPARITÉ DE TRAITEMENT ENTRE SALARIÉS DU MÊME ÉTABLISSEMENT}

Au cours des dernières décennies, le législateur a été amené à se préoccuper des écarts de salaires et autres conditions de travail applicables aux différentes catégories de salariés effectuant le même travail dans un même établissement. La Loi sur les normes du travail prohibait déjà toute différence dans le taux de salaire ou dans la durée du congé annuel défavorable à un salarié parce qu'il travaille moins d'heures que les autres. Les derniers amendements étendent cette interdiction aux écarts fondés sur le statut de travail, visant ainsi le traitement défavorable dont sont souvent victimes les travailleurs au statut atypique. De la même façon, avait déjà été interdite la clause de disparité de traitement que pouvait contenir une convention collective, si celle-ci établissait des conditions de travail moins avantageuses pour les salariés embauchés après une date déterminée parmi ceux exécutant les mêmes tâches dans le même établissement. L'interdiction vise aussi aujourd'hui les régimes de retraite et les avantages sociaux. Ces mesures sont particulièrement significatives en ce qu'elles ont pour effet, au nom de l'intérêt public, de retirer toutes dispositions contenant ces caractéristiques du champ de la négociation collective.

\section{V - LE HARCÈLEMENT PSYCHOLOGIQUE}

Depuis 2002, la Loi sur les normes du travail prescrit que tout salarié a droit à un milieu de travail exempt de harcèlement psychologique qu'elle définit comme: "une conduite vexatoire se manifestant soit par des comportements, des paroles, des actes ou des gestes répétés, qui sont hostiles ou non désirés, laquelle porte atteinte à la dignité ou à l'intégrité psychologique ou physique du salarié et qui entraîne, pour celui-ci, un milieu de travail néfaste ». Bien que la jurisprudence l'eut déjà clairement établi, le législateur, alors que les abus sexuels dont les femmes sont victimes, notamment au travail, faisaient l'objet d'une campagne internationale de dénonciation, ajouta la phrase suivante à cette définition : «Pour plus de précision, le harcèlement psychologique comprend une telle conduite lorsqu'elle se manifeste par de telles paroles, de tels actes ou de tels gestes à caractère sexuel ». II incombe à l'employeur de prendre des moyens raisonnables pour prévenir le harcèlement psychologique et sexuel et, lorsque le phénomène est porté à sa connaissance, de le faire cesser. Les récents amendements apportés à la Loi exigent de plus qu'il établisse une politique de prévention du harcèlement psychologique et de traitement des plaintes, et la rende disponible à ses salariés. Il est spécifiquement mentionné que la politique doit contenir un volet concernant les conduites à caractère sexuel.

\section{VI - LES MESURES FAVORISANT LA CONCILIATION TRAVAIL-FAMILLE}

Plusieurs des récents amendements apportés à la Loi sur les normes du travail s'inscrivent dans la politique visant à favoriser la conciliation travail-famille que poursuit le gouvernement du Québec depuis plus de deux décennies. II s'agit essentiellement de la bonification de normes relatives à cette question qui avaient déjà été introduites dans la Loi. Les principaux changements peuvent être ainsi présentés:

- Le droit à un congé annuel rémunéré d'un minimum de trois semaines est acquis après trois ans de service continu chez le même employeur, au lieu de cinq ans auparavant. 
- Le droit de refuser de travailler plus de quatre heures au-delà des heures quotidiennes habituelles passe à deux heures. Ce même droit vaut désormais lorsque le salarié n'a pas été informé au moins cinq jours à l'avance qu'il serait requis de se présenter au travail.

- L'accès au congé accordé pour raison de maladie, accident ou don d'organe ou de tissus à des fins de greffe, d'une durée maximale de 26 semaines sur une période de 12 mois, est facilité par le retrait de l'exigence de trois mois de service continu chez le même employeur. De plus, les deux premières journées de ce congé sont dorénavant rémunérées, mais seulement si le salarié a accumulé trois mois de service continu ou plus auprès de son employeur.

- Le droit à ce dernier congé est maintenant étendu aux salariés victimes de violence conjugale ou de violence à caractère sexuel.

- La notion de "parent» pour bénéficier du droit à un congé maximum de 10 jours par année pour des obligations liées à la garde, la santé ou l'éducation de son enfant ou d'un proche parent est considérablement élargie. Au conjoint du salarié, à son père, sa mère, un frère, une sœur ou un de ses grands-parents ou de ceux de son conjoint, s'ajoutent plusieurs autres personnes, comme le conjoint de toutes les personnes précitées, leurs enfants et les conjoints de leurs enfants, l'enfant pour lequel le salarié ou son conjoint agit comme famille d'accueil ou la personne envers laquelle le salarié est désigné comme tuteur, curateur ou proche aidant. De plus, les deux premières journées prises annuellement de ce congé qui est autrement sans solde, sont dorénavant rémunérées dès que le salarié justifie de trois mois de service continu auprès de son employeur.

- Ces dernières mesures s'appliquent aussi au droit de s'absenter pour une période prolongée pour s'occuper de son enfant ou d'un parent atteint d'une maladie grave ou qui a subi un grave accident. La durée de ce congé est de plus allongée, passant de 12 à 16 semaines au cours d'une même année, et à 36 semaines s'il s'agit d'un enfant mineur.

- Le droit à un congé d'au plus 27 semaines sans solde au cours d'une période de 12 mois est nouvellement reconnu, lorsque le " parent » (tel que dorénavant défini) du salarié ou son enfant majeur est atteint d'une maladie très grave, qu'un certificat médical atteste potentiellement mortelle, et que son état de santé le requiert.

Aucune des dernières modifications apportées à la Loi sur les normes du travail du Québec n'est en soi très ambitieuse. Elles demeurent généralement timides, et ne correspondent certes pas à la profonde réforme du droit du travail que nécessitent l'évolution du marché du travail et la précarisation généralisée des conditions de travail. Considérées dans leur ensemble toutefois, elles s'avèrent significatives en ce qu'elles visent, parfois pour la première fois, certains des problèmes les plus difficiles que connaissent actuellement les travailleurs salariés au Québec. Tel est le cas des mesures introduites pour s'appliquer spécifiquement aux agences de placement de personnel, aux travailleurs étrangers temporaires et aux agences qui les recrutent. L'accès à un congé, dont les deux premiers jours sont rémunérés, aux salariés victimes de violence conjugale ou à caractère sexuel, est aussi à mentionner. Au-delà de ces mesures concrètes, le fait que le législateur reconnaisse explicitement le phénomène, comme aussi l'inclusion du harcèlement à caractère sexuel dans le harcèlement psychologique, et son effet sur la personne du salarié est en soi une avancée à souligner.

Ces nouvelles mesures confirment par ailleurs le changement d'attitude du législateur à l'égard de la négociation collective. D’une part, elle ne représente plus le véhicule privilégié par l'État pour déployer ses politiques sociales. Le caractère limité de son étendue exige dorénavant de recourir à la législation pour la mise en place de politiques à vocation universelle. D'autre part, ces mesures trahissent un doute quant à la capacité des parties de négocier, dans tous les cas, des conditions de travail jugées justes ou acceptables. L'interdiction des clauses de disparité de traitement selon la date d'embauche ou le statut de travail, dont sont victimes les salariés souvent parmi les plus vulnérables, en témoigne éloquemment. 